\title{
Erratum to: Comparative genomic analysis of mammalian NKG2D ligand family genes provides insights into their origin and evolution
}

\author{
Mizuho Kondo • Takako Maruoka • Noriyuki Otsuka • \\ Jun Kasamatsu • Kazunori Fugo • Naoto Hanzawa • \\ Masanori Kasahara
}

Published online: 25 August 2010

(C) Springer-Verlag 2010

Erratum to: Immunogenetics

DOI 10.1007/s00251-010-0438-z

The original version of this article unfortunately contained a mistake. The presentation of Fig. 4 is incorrect. The corrected figure is given below.

The online version of the original article can be found at http://dx.doi. org/10.1007/s00251-010-0438-z.

\footnotetext{
M. Kondo $\cdot$ T. Maruoka $\cdot$ N. Otsuka $(\bowtie) \cdot J$. Kasamatsu •

K. Fugo $\cdot$ M. Kasahara $(\bowtie)$

Department of Pathology,

Hokkaido University Graduate School of Medicine,

North-15 West-7,

Sapporo 060-8638, Japan

e-mail: tsuka-p@med.hokudai.ac.jp

e-mail: mkasaha@med.hokudai.ac.jp

M. Kondo $\cdot$ N. Hanzawa

Graduate School of Science and Engineering,

Yamagata University,

Yamagata 990-8560, Japan
} 


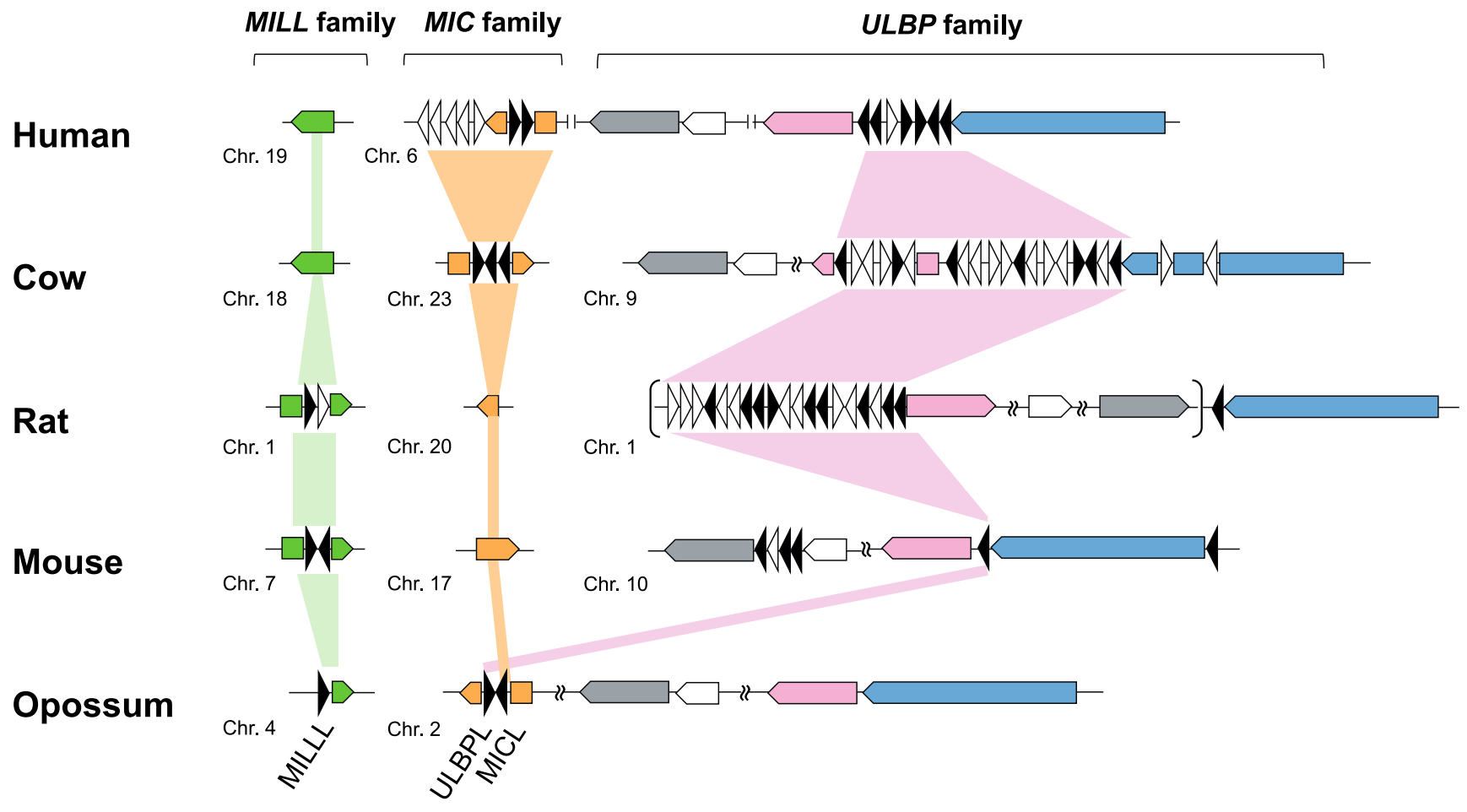

NKG2DL and NKG2DL-like genes

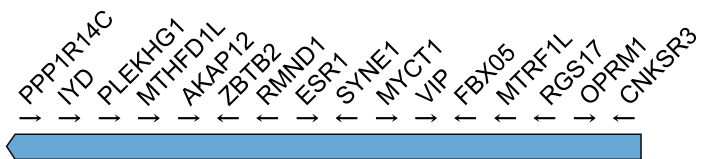

Functional or potentially

functional genes

$\downarrow$ Pseudogenes

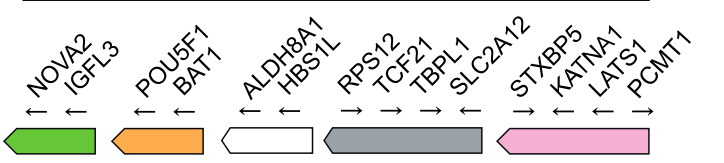

Fig. 4 Comparative maps of NKG2DL and NKG2DL-like gene clusters in mammals. NKG2DL and NKG2DL-like genes are indicated by triangles. Filled and open triangles indicate apparently functional genes and pseudogenes, respectively. Conserved synteny blocks in the vicinity of NKG2DL and NKG2DL-like genes are represented by color-coded boxes. Blue boxes include the following genes: PPP1R14C (protein phosphatase 1 regulatory subunit 14C), IYD (iodotyrosine deiodinase 1 precursor), PLEKHG1 (pleckstrin homology domain containing, family $G$ member 1), MTHFD1L (methylenetetrahydrofolate dehydrogenase 1-like), AKAP12 (A-kinase anchor protein 12), ZBTB2 (zinc finger and BTB domain containing 2), RMND1 (required for meiotic nuclear division 1 homolog), ESR1 (estrogen receptor 1), SYNE1 (synaptic nuclear envelope 1), MYCT1 (myc target 1), VIP (vasoactive intestinal polypeptide), FBXO5 (F-box protein 5), MTRF1L (mitochondrial translational release factor 1-like), RGS17 (regulator of G-protein signaling 17), OPRM1 (opioid receptor, mu 1), and CNKSR3 (connector enhancer of kinase suppressor of ras 3). The order of genes in the blue box is conserved in all species except cattle where the chromosomal segment containing three genes, FBXO5, MTRF1L, and RGS17, is inverted. NOVA2 (neuro-oncological ventral antigen 2) and IGFL3 (insulin growth factor-like family member precursor) are represented by green boxes. Orange boxes include POU5F1 (POU class 5 homeobox 1) and BAT1 (HLA-B associated transcript 1). ALDH8A1 (aldehyde dehydrogenase 8 family, member A1) and HBS1L (HBS1-like) are included in white boxes. RPS12 (ribosomal protein S12), TCF21 (transcription factor 21), TBPL1 (TATA box binding protein-like 1), and SLC2A12 (solute carrier family 2, member 12) are indicated by gray boxes. STXBP5 (syntaxin-binding protein 5), KATNA1 (katanin p60 subunit A1), LATS1 (large tumor suppressor, homolog 1 (Drosophila)), and PCMT1 (protein-L-isoaspartate (D-aspartate) O-methyltransferase 1) are represented by pink boxes. Genes were named according to the nomenclature of human genes. In the opossum genome, IGFL3 was not identified presumably because the genome assembly was incomplete. In the rat genome, POU5F1 was not identified 